\title{
Modelling isotropic and kinematic hardening of granular materials with a thermodynamical approach
}

\author{
L. F. Miranda Pino ${ }^{1}$, Piaras A. Kelly ${ }^{2}$ and Beatrice A. Baudet ${ }^{3}$ \\ ${ }^{1}$ Department of Civil Engineering, The University of Hong Kong, Pokfulam, Hong \\ Kong, China; email: felipe.miranda@hku.hk \\ ${ }^{2}$ Department of Engineering Science, University of Auckland, Private Bag 93029,Auck- \\ land, New Zealand; email: pa.kelly@ auckland.ac.nz \\ ${ }^{3}$ Department of Civil, Environmental and Geomatic Engineering, University College \\ London, U.K., formerly The University of Hong Kong; email: b.baudet @ucl.ac.uk
}

\begin{abstract}
Thermodynamics based models for granular and porous materials have been widely used in the development of more complex models. Broadly speaking, there exists two approaches; in both, the first law of thermodynamics enables one to model the recoverable response. However, the approaches differ slightly when dealing with the second law of thermodynamics, i.e. irreversable processes. The French school approach introduces the notion of thermodynamic forces, flow and evolution rules. Ziegler's approach directly postulates a positive dissipation function on a dissipative stress space, which then requires an extra mapping step to the true stress space. Despite the fact that they both have the same foundations, differences emerge between them regarding the way in which stored energy is accounted for in the development of kinematic and/or isotropic hardening. In Ziegler's approach, the stored energy is solely used for the kinematic hardening, as opposed to the French school approach in which the stored energy is allowed in both cases of hardening. Despite which modelling approach is taken, we will show how certain modifications to these theories have to be made in order to develop classical models.
\end{abstract}

\section{BACKGROUND}

The dissipation function for isothermal processes which are only subject to small strains is defined by the Clausius - Plank inequality:

$$
\dot{\Phi}=\sigma_{i j} \dot{\varepsilon}_{i j}-\dot{\Psi} \geq 0
$$

Where $\dot{\Phi}$ is the rate of dissipation, $\sigma_{i j}$ is the Cauchy stress tensor, the small strain tensor is $\varepsilon_{i j}$ and $\dot{\Psi}$ the rate of Helmholtz free energy .

\section{Reversible process}

For a reversible process there exists no dissipation and all the work done can be retrieved after the process ends. In such instances, the state of the material is completely defined by its observable variables $\left(\theta, \varepsilon_{i j}\right)$, where $\theta$ is the temperature, omitted 
in this study. The rate of the free energy is solely a function of strain $\left(\Psi\left(\varepsilon_{i j}\right)\right)$. Since the dissipation rate is zero for reversible processes, it follows:

$$
\left(\sigma_{i j}-\frac{\partial \Psi}{\partial \varepsilon_{i j}}\right) \dot{\varepsilon}_{i j}=0
$$

For a non viscous material (i.e. $\sigma_{i j} \neq \sigma_{i j}\left(\dot{\varepsilon}_{i j}\right)$ ), the terms in the parenthesis are independent of the strain rate, thus:

$$
\sigma_{i j}=\frac{\partial \Psi}{\partial \varepsilon_{i j}}
$$

\section{Irreversible process}

When dealing with irreversible processes, the dissipation rate is no longer zero; furthermore, a new set of state variables must be introduced. These variables are often termed internal variables, because, as opposed to the state variables previously discussed, they can not be measured in a straight forward manner. These internal variables are often strain-like variables such as plastic strains (but not only), which are the focus of this study. At this time, two approaches have emerged to ensure that the Clausius Plank inequality (1) holds. These are: the French school approach, the one introduced in the classic books of Lemaitre and Chaboche (1994) and Coussy (2004), and the approach which makes use of Ziegler's postulation, hereafter termed Ziegler's approach; accounts for this latter approach could be found in, for example, the paper by Collins and Houlsby (1997).

\section{French school approach}

The free energy is now required to be a function of observable variables $\left(\varepsilon_{i j}\right)$ and internal variables $\left(\varepsilon_{i j}^{p}, \xi_{i}\right)$, which could also be decoupled into elastic and plastic parts under the small strain theory.

$$
\Psi\left(\varepsilon_{i j}-\varepsilon_{i j}^{p}, \xi_{i}\right)=\Psi^{e}\left(\varepsilon_{i j}-\varepsilon_{i j}^{p}\right)+\Psi^{p}\left(\xi_{i}\right)
$$

The inequality (1) can be re-arranged as follows:

$$
\left(\sigma_{i j}-\frac{\partial \Psi}{\partial \varepsilon_{i j}}\right) \dot{\varepsilon}_{i j}+\frac{\partial \Psi}{\partial \varepsilon_{i j}} \dot{\varepsilon}_{i j}^{p}-\frac{\partial \Psi^{p}}{\partial \xi_{i}} \dot{\xi}_{i} \geq 0
$$

It can be argued, even under irreversible processes, that the term inside the parenthesis must be zero (Coleman and Gurtin 1967). Hence, the inequality reduces to the following expression:

$$
\dot{\Phi}=\sigma_{i j} \dot{\varepsilon}_{i j}^{p}-\chi_{i} \dot{\xi}_{i} \geq 0
$$

where the stress and the thermodynamics forces $\left(\chi_{i}\right)$ are:

$$
\sigma_{i j}=\frac{\partial \Psi}{\partial \varepsilon_{i j}} ; \quad \chi_{i}=\frac{\partial \Psi^{p}}{\partial \xi_{i}}
$$

In other to ensure that the inequality (4) holds, both terms are enforced to be positive by introducing a convex potential $\left(g\left(\sigma_{i j}, \chi_{i}\right)\right)$. The expression for the rate of plastic strain 
is known as the flow rule and the one for the internal variables the evolution rule. The positiveness of the dissipation holds for a convex potential function $(g)$ whose minimum lies at the origin.

$$
\dot{\varepsilon}_{i j}^{p}=\dot{\lambda} \frac{\partial g}{\partial \sigma_{i j}} ; \quad \dot{\xi}_{i}=-\dot{\lambda} \frac{\partial g}{\partial \chi_{i}}
$$

\section{Ziegler's approach}

The observable variable is the total strain $\left(\varepsilon_{i j}\right)$ and the set of internal variables are termed $\alpha_{i j}$ which could include the plastic strains. Therefore, the free energy and dissipation function are defined as:

$$
\Psi=\Psi\left(\varepsilon_{i j}, \alpha_{i j}\right) ; \quad \dot{\Phi}=\dot{\Phi}\left(\varepsilon_{i j}, \alpha_{i j}, \dot{\alpha}_{i j}\right)
$$

The dissipation function for rate independent materials must be a homogeneous function of degree one in the rates since there is no characteristic time. From Euler's theorem for homogeneous functions:

$$
\dot{\Phi}=\frac{\partial \dot{\Phi}}{\partial \dot{\alpha}_{i j}} \dot{\alpha}_{i j}
$$

Using the dissipation inequality (1):

$$
\left(\frac{\partial \dot{\Phi}}{\partial \dot{\alpha}_{i j}}+\frac{\partial \Psi}{\partial \alpha_{i j}}\right) \dot{\alpha}_{i j}=\left(\sigma_{i j}-\frac{\partial \Psi}{\partial \varepsilon_{i j}}\right) \dot{\varepsilon}_{i j}
$$

Since both observable and internal variables are independent of each other both sides of this equation must be zero. The right side of the equation is clearly independent of the strain rate, thus equal to zero:

$$
\sigma_{i j}=\frac{\partial \Psi}{\partial \varepsilon_{i j}}
$$

However, the left side of the equation is dependent on the rate of internal variables, thus one cannot proceed as previously. The principle of maximum dissipation was invoked by Ziegler in order to satisfy the equation, in which case the left side is assumed to equal to zero:

$$
\frac{\partial \dot{\Phi}}{\partial \dot{\alpha}_{i j}}=-\frac{\partial \Psi}{\partial \alpha_{i j}}
$$

This equation is also known as Ziegler's postulate, where the dissipative stress $\left(\sigma_{i j}^{d}\right)$ and the quasi-conservative stress $\left(\bar{\sigma}_{i j}\right)$ are defined to be:

$$
\sigma_{i j}^{d}=\frac{\partial \dot{\Phi}}{\partial \dot{\alpha}_{i j}} ; \quad \bar{\sigma}_{i j}=-\frac{\partial \Psi}{\partial \alpha_{i j}}
$$

To ensure the positiveness of the dissipation function, it itself is postulated to be a convex potential of the rate of internal variables, similar to the case for the potential $g$. Moreover, to eliminate the rate of internal variables $\left(\dot{\alpha}_{i j}\right)$ from this function, the Legendre transform of the dissipation is taken. Since it was already note that the the 
dissipation function is a homogeneous function of degree one in the rate of internal variables, this Legendre transform is singular (i.e. equal to zero).

$$
w\left(\sigma_{i j}^{d}\right)=\dot{\alpha}_{i j} \sigma_{i j}^{d}-\dot{\Phi}\left(\dot{\alpha}_{i j}\right)=0 ; \quad \text { where: } \quad \dot{\alpha}_{i j}=\frac{\partial w}{\partial \sigma_{i j}^{d}} ; \quad \sigma_{i j}^{d}=\frac{\partial \dot{\Phi}}{\partial \dot{\alpha}_{i j}}
$$

By taking the Legendre transform (9) the dissipative stress has the same expression as in (8). Since the function $w$ is equal to zero it can be determined to within an undetermined multiplier $(\dot{\lambda})$ :

$$
w\left(\sigma_{i j}^{d}\right)=\dot{\lambda} F\left(\sigma_{i j}^{d}\right)
$$

This function $F$ represents the yield function on the dissipative stress space; from (9) the internal variable is:

$$
\alpha_{i j}=\dot{\lambda} \frac{\partial F}{\partial \sigma_{i j}^{d}}
$$

Note that the internal variable is normal (i.e associated) to the yield function in dissipative stress space, which is a result of the Legendre transform performed in (9). In the case of a decoupled response, the free energy could be decomposed, similarly to (3); thus the quasi-conservative stress from (8) is:

$$
\bar{\sigma}_{i j}=-\left(\frac{\partial \Psi^{e}}{\partial \alpha_{i j}}+\frac{\mathrm{d} \Psi^{p}}{\mathrm{~d} \alpha_{i j}}\right)
$$

Define, the shift stress $\left(\sigma_{i j}^{s}\right)$ :

$$
\sigma_{i j}^{s}=\frac{\mathrm{d} \Psi^{p}}{\mathrm{~d} \alpha_{i j}}
$$

Using Ziegler's postulate (7), the dissipative stress is then:

$$
\sigma_{i j}^{d}=\bar{\sigma}_{i j}=\sigma_{i j}-\sigma_{i j}^{s}
$$

This equation is used to map the yield function from the dissipative stress space $(F)$ into the true stress space, thus defining the yield function in true stress space $(f)$ :

$$
f\left(\sigma_{i j}-\sigma_{i j}^{s}\right)=0
$$

\section{DISCUSSION}

Under the assumption of a decoupled response (3), the free energy function was divided into its elastic and plastic components. The elastic component represents the energy that can be recovered after loading has been retrieved, and the stress is derived from it in the same manner as in the hyperelastic theory. The plastic component of the free energy is also termed frozen energy or stored energy, and allows the introduction of the thermodynamic force and the shift stress, under the French school approach and Ziegler's approach, respectively. The implications of the stored energy on each approach are discussed below by applying them to classical models. 


\section{Linear kinematic hardening - Von Mises 1D model}

First, the case of kinematic hardening will be examined.

\section{French school approach}

According to this approach two functions are to be postulated: Free energy $(\Psi)$ and a convex potential $(g)$.

$$
\Psi=\Psi^{e}\left(\varepsilon-\varepsilon^{p}\right)+\Psi^{p}(\xi)=\frac{E}{2}\left(\varepsilon-\varepsilon^{p}\right)^{2}+\frac{1}{2} H \xi^{2} ; \quad g=f=|\sigma-\chi|-Y_{o}=0
$$

Where $E$ is the Young's modulus and the yield stress is $Y_{o}$, the stress and thermodynamic force are:

$$
\sigma=\frac{\partial \Psi}{\partial \varepsilon}=E\left(\varepsilon-\varepsilon^{p}\right) ; \quad \chi=\frac{\partial \Psi^{p}}{\partial \chi}=H \xi
$$

Notice that in this approach we are yet to define the internal variable $(\xi)$. The evolution rule and flow rule are:

$$
\dot{\varepsilon}^{p}=\dot{\lambda} \frac{\partial g}{\partial \sigma}=\dot{\lambda} \operatorname{sgn}(\sigma-\chi) ; \quad \dot{\xi}=-\dot{\lambda} \frac{\partial g}{\partial \chi}=\dot{\lambda} \operatorname{sgn}(\sigma-\chi)=\dot{\varepsilon}^{p}
$$

An associated flow rule was considered (i.e $g=f$ ). This means that the direction of stress and plastic strain rate are identical in one dimension; this could be expressed as: $\left(\operatorname{sgn}(\sigma-\chi)=\operatorname{sgn}\left(\dot{\varepsilon}^{p}\right)\right)$, which in addition to (4) yields the rate of dissipation:

$$
\dot{\Phi}=(\sigma-\chi) \dot{\varepsilon}^{p}=Y_{o}\left|\dot{\varepsilon}^{p}\right|
$$

Ziegler's approach

In this case the functions to be postulated are: Free energy and the rate of dissipation function:

The stresses are:

$$
\Psi=\frac{E}{2}\left(\varepsilon-\varepsilon^{p}\right)^{2}+\frac{1}{2} H\left(\varepsilon^{p}\right)^{2} ; \quad \dot{\Phi}=Y_{o}\left|\dot{\varepsilon}^{p}\right|
$$

$$
\sigma=\frac{\partial \Psi}{\partial \varepsilon}=E\left(\varepsilon-\varepsilon^{p}\right) ; \quad \sigma_{s}=\frac{\partial \Psi^{p}}{\partial \varepsilon^{p}}=H \varepsilon^{p} ; \quad \sigma_{d}=\frac{\partial \dot{\Phi}}{\partial \varepsilon^{p}}=Y_{o} \operatorname{sgn}\left(\dot{\varepsilon}_{p}\right)
$$

Eliminating the rate of the internal variable from the expression of the dissipative stress yields the yield function, which was achieved by the Legendre transform (9). According to (10), in dissipative space the associate flow rule holds (i.e $\operatorname{sgn}\left(\dot{\varepsilon}^{p}\right)=\operatorname{sgn}\left(\sigma_{d}\right)$ ); thus, the yield function on dissipative stress is:

$$
F=\left|\sigma_{d}\right|-Y_{o}=0
$$

And from (11), the yield function in true stress space $(f)$ :

$$
f=\left|\sigma-\sigma_{s}\right|-Y_{o}=0
$$

The rate of the internal variable is:

$$
\dot{\varepsilon}^{p}=\dot{\lambda} \frac{\partial F}{\partial \sigma_{d}}=\dot{\lambda} \operatorname{sgn}\left(\sigma_{d}\right)=\dot{\lambda} \operatorname{sgn}\left(\sigma-\sigma_{s}\right)
$$

From the above, we conclude that, when kinematic hardening is modeled, the mechanical responses as derived using both approaches are identical. Moreover, the stored energy and dissipation are exactly the same in both cases as well. 


\section{Linear isotropic hardening - Von Mises 1D model}

Next, the case of isotropic hardening will be examined.

French school approach

The free energy $(\Psi)$ and a convex potential $(g)$ are postulated:

$$
\Psi=\frac{E}{2}\left(\varepsilon-\varepsilon^{p}\right)^{2}+\frac{1}{2} H \xi^{2} ; \quad g=f=|\sigma|-\chi-Y_{o}=0
$$

The stresses and thermodynamic force are:

$$
\sigma=\frac{\partial \Psi}{\partial \varepsilon}=E\left(\varepsilon-\varepsilon^{p}\right) ; \quad \chi=\frac{\partial \Psi^{p}}{\partial \chi}=H \xi
$$

The evolution rule and flow rule are:

$$
\dot{\varepsilon}^{p}=\dot{\lambda} \operatorname{sgn}(\sigma) ; \quad \dot{\xi}=\dot{\lambda}
$$

Since we are considering associated flow rule (i.e $g=f$ ) the direction of the stress and the plastic strain rate are the same, thus:

$$
\dot{\xi}=\left|\dot{\varepsilon}^{p}\right|
$$

\section{Ziegler's approach}

Here, let the free energy and the rate of dissipation function be:

$$
\Psi=\frac{E}{2}\left(\varepsilon-\varepsilon^{p}\right)^{2} ; \quad \dot{\Phi}=\left(Y_{o}+H \varepsilon^{p}\right)\left|\dot{\varepsilon}^{p}\right|
$$

The stresses are:

$$
\sigma=E\left(\varepsilon-\varepsilon^{p}\right) ; \quad \sigma_{s}=0 ; \quad \sigma_{d}=\left(Y_{o}+H \varepsilon^{p}\right) \operatorname{sgn}\left(\dot{\varepsilon}^{p}\right)
$$

We proceed identically as before, eliminating the rate of internal variable from the dissipation function. Furthermore, since the shift stress is zero, the yield functions in both spaces have the same expression:

$$
F=\left|\sigma_{d}\right|-H \varepsilon^{p}-Y_{o}=0 ; \quad f=|\sigma|-H \varepsilon^{p}-Y_{o}=0
$$

The rate of the internal variable is:

$$
\dot{\varepsilon}^{p}=\dot{\lambda} \operatorname{sgn}\left(\sigma_{d}\right)=\dot{\lambda} \operatorname{sgn}(\sigma)
$$

It is clear that the mechanical responses derived by these two approaches are identical; however, unlike to the case of kinematic hardening, differences emerge in the expressions for the stored energy and the dissipation rate. The French school approach, as for kinematic hardening, considers that there exists a part of the plastic work done to be transformed into stored energy (i.e. $\Psi^{p} \neq 0$ ), and the rest is dissipated. The stored 
energy in theory could be retrieved under unloading; however, we observe that the internal variable rate $(\dot{\xi})$ is equal to the absolute value of the plastic strain rate. Hence, the internal variable rate under any loading condition is always positive. Therefore, the stored energy always accumulates and it cannot be retrieved, as opposed to the case of kinematic hardening in which the stored energy is recovered (Besson et al. 2009). In contrast, in Ziegler's approach, there is no stored energy involved in isotropic hardening (i.e all the plastic work done is dissipated). Such a condition in the French school approach is only observed in rigid hardening (Ottosen and Ristinmaa 2005). It is also worth noting that the dissipation function used in the isotropic case is also dependent on the plastic strain, not only its rate. The hierarchy of plastic models for both approaches is presented in Table 1, which has been partially modified from Houlsby and Puzrin (2007).

Table 1. Hierarchy of plastic models

\begin{tabular}{llll}
\hline \multicolumn{1}{c}{ Approach } & \multicolumn{1}{c}{ French school } & \multicolumn{2}{c}{ Ziegler's } \\
\hline \hline Hardening type & \multicolumn{1}{c}{ Free energy } & \multicolumn{1}{c}{ Free energy } & Dissipation \\
\hline Perfectly Plastic & $\Psi^{e}\left(\varepsilon_{i j}-\varepsilon_{i j}^{p}\right)$ & $\Psi^{e}\left(\varepsilon_{i j}-\alpha_{i j}\right)$ & $\dot{\Phi}\left(\dot{\alpha}_{i j}\right)$ \\
Isotropic & $\Psi^{e}\left(\varepsilon_{i j}-\varepsilon_{i j}^{p}\right)+\Psi^{p}\left(\xi_{i}\right)$ & $\Psi^{e}\left(\varepsilon_{i j}-\alpha_{i j}\right)$ & $\dot{\Phi}\left(\alpha_{i j}, \dot{\alpha}_{i j}\right)$ \\
Kinematic & $\Psi^{e}\left(\varepsilon_{i j}-\varepsilon_{i j}^{p}\right)+\Psi^{p}\left(\xi_{i}\right)$ & $\Psi^{e}\left(\varepsilon_{i j}-\alpha_{i j}\right)+\Psi^{p}\left(\alpha_{i j}\right)$ & $\dot{\Phi}\left(\dot{\alpha}_{i j}\right)$ \\
Mixed & $\Psi^{e}\left(\varepsilon_{i j}-\varepsilon_{i j}^{p}\right)+\Psi^{p}\left(\xi_{i}\right)$ & $\Psi^{e}\left(\varepsilon_{i j}-\alpha_{i j}\right)+\Psi^{p}\left(\alpha_{i j}\right)$ & $\dot{\Phi}\left(\alpha_{i j}, \dot{\alpha}_{i j}\right)$ \\
\hline
\end{tabular}

\section{Modified cam-clay model (MCC)}

The modified cam-clay model is probably the single most important model in soil mechanics as it has been widely used in the development of many advanced models. Although initially intended for fully saturated materials it has also been extended to partially saturated materials. As is well known, MCC is a plastic volumetric strain isotropic hardening model, i.e the plastic volumetric strain $\left(\varepsilon_{v}^{p}\right)$ is the hardening variable, and the preconsolidation pressure $\left(p_{c}\right)$ is the hardening parameter.

\section{French school approach}

As stated above the hardening parameter is $p_{c}$ which under this approach is equivalent to the thermodynamic force $\left(\chi=p_{c}\right)$. Furthermore, the internal variable now is set to be the plastic volumetric strain $\left(\xi=\varepsilon_{v}^{p}\right)$, thus $p_{c}\left(\varepsilon_{v}^{p}\right)$. The free energy and the potential function with an associated flow rule are:

$$
\Psi=\Psi^{e}+\frac{1}{2} \int p_{c}\left(\varepsilon_{v}^{p}\right) \mathrm{d} \varepsilon_{v}^{p} ; \quad g=f=q^{2}+M^{2} p^{2}-M^{2} p p_{c}=0
$$

Let us focus on the flow rule and evolution rule:

$$
\dot{\varepsilon}_{v}^{p}=\dot{\lambda} \frac{\partial g}{\partial p}=\dot{\lambda} M^{2}\left(2 p-p_{c}\right) ; \quad \dot{\xi}=-\dot{\lambda} \frac{\partial g}{\partial p_{c}}=\dot{\lambda} M^{2} p
$$

By no means can the expressions for the rate of volumetric plastic strain and the internal variable be equal. Furthermore, there is no expression for $g$ that would satisfy: $\xi=\varepsilon_{v}^{p}$; 
and at the same time satisfy the associated flow rule. Under this circumstance Ulm and Coussy (2003, p. 282) indicated that it is not always required to postulate the same convex function for both flow rule and evolution rule. Independent functions (e.g. $g$ and $h$ ) could be used, so long as that they are convex with respect to their argument(s) to ensure the positiveness of (1). In this way one could have an associated flow rule $(g=f)$ and non-associated hardening rule $(h \neq f)$. Based on this, the new function $h$ is required to be:

$$
\dot{\xi}=-\dot{\lambda} \frac{\partial h}{\partial p_{c}} \equiv \dot{\varepsilon}_{v}^{p}=\dot{\lambda} M^{2}\left(2 p-p_{c}\right) \Longrightarrow h=\frac{M^{2}}{2}\left(2 p-p_{c}\right)^{2}
$$

And the governing equations are now:

$$
\Psi=\Psi^{e}+\frac{1}{2} \int p_{c}\left(\varepsilon_{v}^{p}\right) \mathrm{d} \varepsilon_{v}^{p} ; \quad g=f=q^{2}+M^{2} p^{2}-M^{2} p p_{c}=0 ; \quad h=\frac{M^{2}}{2}\left(2 p-p_{c}\right)^{2}
$$

Ziegler's approach

The dissipation function and free energy are:

$$
\Psi=\Psi^{e}+\frac{1}{2} \int p_{c}\left(\varepsilon_{v}^{p}\right) \mathrm{d} \varepsilon_{v}^{p} ; \quad \Phi=\frac{1}{2} p_{c}\left(\varepsilon_{v}^{p}\right)\left(\sqrt{\left(\dot{\varepsilon}_{v}^{p}\right)^{2}+M^{2}\left(\dot{\varepsilon}_{s}^{p}\right)^{2}}\right)
$$

Collins and Houlsby (1997) showed that in order to be able to develop the modified Cam-clay model it is required to use a shift stress such that it will "shift" the yield surface in the deviatoric - mean effective stress plane $(p-q)$ to the origin. They also showed that it was possible to generate it, simply by adding the stored energy to the dissipation function. In any case; however, not all the plastic work is dissipated, a fraction of it will be stored. This appears not to be inline with the general theory for Ziegler's approach which states that for an isotropic hardening model all the plastic work is dissipated, thus, the stored energy is zero. The governing equations presented above resemble the case of mixed hardening instead of the isotropic hardening as presented in table 1.

\section{CONCLUSION}

Two widely used approaches which apply the laws of thermodynamics in continuum mechanics have been examined in the context of classical models. On the outline the French school's approach is simpler as it resembles more to the classical elastoplastic theory whereas in Ziegler's approach the use of Legendre transforms adds some extra difficulties. Conceptually, there are two main differences worth noting when adopting one or the other approach in the development of constitutive models for soils: a) how the stored energy is used and b) how the yield function is defined. For example, while in the French school's approach stored energy is generated whatever the type of hardening (i.e. isotropic or kinematic), in Ziegler's approach only energy is only stored during kinematic hardening. The distinct approaches also lead to different ways to define the yield function, which is derived from the dissipation function in the Ziegler's approachs case, while in the French school's approach it is usually simply taken equal to the potential function, the only restriction being that this potential function should be convex. Applying the different approaches to developing the classical Modified Cam 
Clay model further shows that it cannot be done without modification either in the form for a non-associated evolution rule, or by using a mixed hardening model, thus highlighting the deviation of the classical model from thermodynamics rules.

\section{REFERENCES}

Besson, J., Cailletaud, G., Chaboche, J.-L., and Forest, S. (2009). Non-linear mechanics of materials. Springer Science \& Business Media.

Coleman, B. D. and Gurtin, M. E. (1967). "Thermodynamics with internal state variables". The Journal of Chemical Physics, 47(2), 597613.

Collins, I. F. and Houlsby, G. T. (1997). "Application of thermomechanical principles to the modelling of geotechnical materials". In Proceedings of the royal society of london a: mathematical, physical and engineering sciences (Vol. 453, 1964, pp. 19752001). The Royal Society.

Coussy, O. (2004). Poromechanics. J. Wiley \& Sons.

Houlsby, G. T. and Puzrin, A. M. (2007). Principles of hyperplasticity: an approach to plasticity theory based on thermodynamic principles. Springer Science \& Business Media.

Lemaitre, J. and Chaboche, J.-L. (1994). Mechanics of solid materials. Cambridge university press.

Ottosen, N. S. and Ristinmaa, M. (2005). The mechanics of constitutive modeling. Elsevier.

Ulm, F.-J. and Coussy, O. (2003). Mechanics and durability of solids. Prentice Hall. 